\title{
Successful Search for Ether Drift in a Modified Michelson- Morley Experiment Using the GPS
}

\author{
Stephan J. G. Gift \\ Department of Electrical and Computer Engineering \\ Faculty of Engineering \\ The University of the West Indies \\ St. Augustine, Trinidad and Tobago, West Indies \\ Email: Stephan.Gift@sta.uwi.edu
}

\begin{abstract}
Ether drift resulting from the rotation of the Earth has been detected. This was accomplished using GPS technology in a modified Michelson-Morley experiment. The original Michelson-Morley experiment searched for ether drift by observing round-trip light travel time differences using interference fringe shifts. This method is limited by length contraction effects that significantly reduce any fringe shifts. In the modified approach elapsed time for one-way light transmission is directly determined using GPS clocks. The method yields travel time differences for light transmission in the East-West direction but not in the North-South direction consistent with rotationally-induced ether drift.
\end{abstract}

Keywords: Michelson-Morley experiment, ether drift, GPS, synchronized clocks, one-way speed of light, ECI frame.

\section{Introduction}

One of the most celebrated experiments of all time is the Michelson-Morley experiment of 1887 that searched for ether drift based on interferometer fringe shifts [1]. This experiment involved interfering light beams that traversed orthogonal paths on a movable apparatus. It was designed to reveal the speed of the Earth's orbital motion through the hypothesized ether using the expected change in light speed arising from movement with or against the associated ether wind. Because the light beam was two-way, the experiment was of second-order in that the fringe-shift being searched for was proportional to the second power of the ratio of the Earth's orbital velocity and the velocity of light $c$. The fringe shift observed by Michelson and Morley was significantly less than that expected as a result of the revolving Earth and is generally interpreted as an essentially null result.

A major enhancement of this basic experiment was introduced by T. S. Jaseja et al. in 1964 [2]. These researchers employed laser technology to realize a sensitivity increase of 25 
times the original experiment but detected no change in the system's beat frequency within its measurement accuracy. A later improved version of the Jaseja experiment performed by Brillet and Hall [3] in 1979 searched for light speed anisotropy in the form of changes in the resonant frequency of a cavity resonator. These researchers claimed a 4000-fold improvement on the measurement by Jaseja et al and again detected no change.

Modern versions of the Michelson-Morley experiment developed along the lines of the approach by Brillet and Hall use electromagnetic resonators that examine light speed isotropy. These systems in general compare the resonant frequencies of two orthogonal resonators in response to orbital or rotational movement. Several versions of this modern Michelson-Morley type experiment include those by Muller et al. [4], Wolfe et al. [5], Hermann et al.[6], Antonini et al. [7], Muller et al. [8], Eisele et al. [9] and Hermann et al. [10]. These experiments have progesssively lowered the limit on light speed anisotropy. The most recent of these set an upper limit of $\delta c / c<10^{-17}$, a value many orders of magnitude below the fractional light speed changes that in the presence of an ether would result from the Earth's orbital speed of 29,765m/s $\left(\delta c / c \approx 10^{-4}\right)$ or even its smaller rotational equatorial surface speed of $463.8 \mathrm{~m} / \mathrm{s}\left(\delta c / c \approx 10^{-6}\right)$.

Despite this collection of negative experiments, Demjanov [11] recently reported positive fringe shifts in a Michelson-Morley experiment involving optically dense media. According to the report the fringe shift disappeared when the optically dense medium was replaced by a vacuum because of second-order length contraction of the arm that is parallel to the direction of motion of the apparatus. This, the researcher claimed, explains the approximately null results of the many Michelson-Morley-type experiments [12]. Demjanov also described a first-order Michelson-Morley experiment in which positive fringe shifts were observed [13]. In a related type of experiment employing an optically dense medium, Galaev [14] reported positive fringe shifts in a modified Michelson-Morley experiment. In addition to these findings, positive results for the Earth's orbital motion have been reported in experiments based on the Roemer effect [15], the Doppler effect [15] and Bradley aberration [16]. It appears however that none of these positive results has attracted the attention of the scientific community.

In search of ether-drift resulting from the Earth's rotational rather than orbital motion we employ the basic Michelson-Morley experiment with modification. Instead of the indirect determination of time differences for light travelling out and back along the arms of the 
apparatus using interferometry as was done in the original experiment, we utilize the synchronized clocks of the GPS to directly determine the travel times of one-way light transmission along the arms of the apparatus. The widely used GPS has been rigorously and extensively tested and verified. Its synchronized clocks are employed in a wide range of time measurement applications including time-stamping of business transactions, network synchronization and time transfer. The system has recently been used to accurately maintain clock synchronization in the OPERA experiment at CERN involving the travel time measurement of fast-moving neutrinos [17]. This modern system is therefore very suitable for travel time determination over fixed distances as in our proposed test.

Sardin [18] had previously proposed the direct measurement of the light travel times along the arms of the Michelson-Morley apparatus using LIGO with multiple reflections. This test was never conducted because of the inadequacy of equipment sensitivity. The approach proposed in this paper does not encounter this problem since a novel feature of the method is that the light travel time is directly available from the GPS clock synchronization algorithm adopted by the CCIR which renders actual signal timing completely unnecessary [19]. In principle timeof-flight determination over any distance on the surface of the Earth is possible using this method.

\section{Original Michelson-Morley Experiment [1]}

The basic apparatus of the original Michelson-Morley experiment is shown in fig. 1 where the apparatus is moving with velocity $v$ through the hypothesized ether in direction PM1. Light from a source S splits into two beams at beam-splitter P. One beam travels from P to mirror M1 and back and is reflected at $\mathrm{P}$ into the interferometer $\mathrm{I}$. The second beam is reflected at $\mathrm{P}$ to mirror M2 and back and passes through P into the interferometer I where both beams form an interference pattern. In the frame of the moving apparatus as a result of ether drift, the resultant light speed between $\mathrm{P}$ and M1 would be $c-v$ toward $\mathrm{M} 1$ and $c+v$ toward $\mathrm{P}$ while the resultant light speed between $\mathrm{P}$ and M2 would be $\left(c^{2}-v^{2}\right)^{1 / 2}$ in both directions. For optical path lengths $P M 1=l_{1}$ and $P M 2=l_{2}$ the time $t_{1}(a)$ for the light to travel from $\mathrm{P}$ to $\mathrm{M} 1$ is given by 


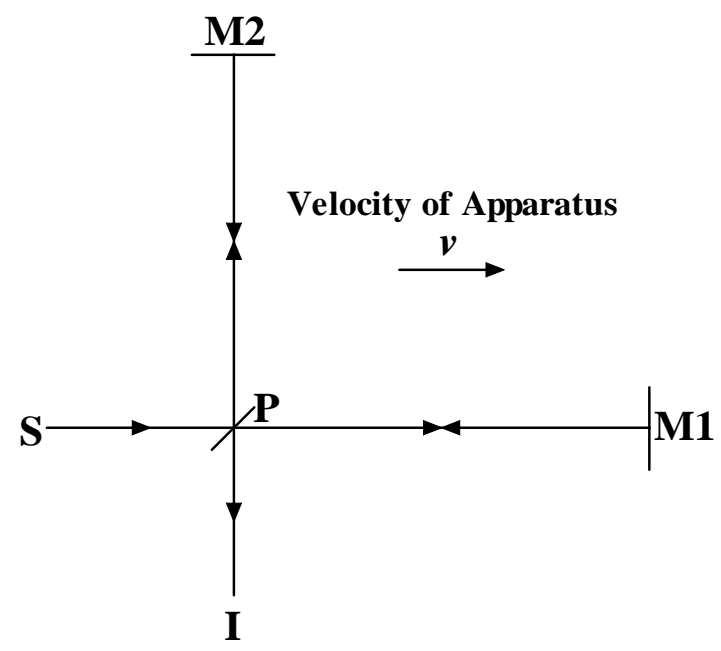

Fig.1 Michelson-Morley Experiment

$$
t_{1}(a)=\frac{l_{1}}{c-v}
$$

and the time $t_{1}(b)$ for the light to travel from M1 to $\mathrm{P}$ is given by

$$
t_{1}(b)=\frac{l_{1}}{c+v}
$$

Therefore the round-trip time along PM1 is given by

$$
T_{1}=t_{1}(a)+t_{1}(b)=\frac{2 l_{1}}{c\left(1-v^{2} / c^{2}\right)}
$$

The time $t_{2}(a)$ for the light to travel from $\mathrm{P}$ to $\mathrm{M} 2$ is given by

$$
t_{2}(a)=\frac{l_{2}}{\sqrt{c^{2}-v^{2}}}
$$

and the time $t_{2}(b)$ for the light to travel from $\mathrm{M} 2$ to $\mathrm{P}$ is given by

$$
t_{2}(b)=\frac{l_{2}}{\sqrt{c^{2}-v^{2}}}
$$

Therefore the round-trip time along PM2 is given by

$$
T_{2}=t_{2}(a)+t_{2}(b)=\frac{2 l_{2}}{c\left(1-v^{2} / c^{2}\right)^{1 / 2}}
$$

The time difference $\Delta T=T_{1}-T_{2}$ is given by 


$$
\Delta T=T_{1}-T_{2} \approx \frac{2\left(l_{1}-l_{2}\right)}{c}+\frac{2 l_{1} v^{2}}{c^{3}}-\frac{l_{2} v^{2}}{c^{3}}
$$

If the apparatus is turned through $90^{\circ}$ so that PM2 is in the direction of motion, the time difference becomes

$$
\Delta T^{\prime}=T_{1}^{\prime}-T_{2}^{\prime} \approx \frac{2\left(l_{1}-l_{2}\right)}{c}+\frac{l_{1} v^{2}}{c^{3}}-\frac{2 l_{2} v^{2}}{c^{3}}
$$

The change in these time differences is

$$
\Delta=\Delta T-\Delta T^{\prime}=\left(l_{1}+l_{2}\right) \frac{v^{2}}{c^{3}}
$$

If $l_{1}=l_{2}=l$ then this reduces to

$$
\Delta=\frac{2 l}{c} \frac{v^{2}}{c^{2}}
$$

A fringe shift proportional to this value (given by $\delta=\frac{c}{\lambda} \Delta$ ) is expected to appear in the interferometer. The time difference $\Delta=\frac{2 l}{c} \frac{v^{2}}{c^{2}}$ is second-order and is significantly reduced by length contraction arising from motion through the ether [11, 12]. This is why Michelson-Morley type experiments have been largely unsuccessful in detecting ether drift.

\section{Modified Michelson-Morley Experiment}

The availability of accurate synchronized clocks in the GPS allows the direct determination of one-way light travel. Thus in a modification of the original Michelson-Morley apparatus GPS clocks are placed at P, M1 and M2 in fig.1. Additionally the arm PM1 is oriented along a line of latitude and the arm PM2 is positioned along a line of longitude. As a result of the rotation of the Earth there is movement of the apparatus at velocity $v=w$ in the direction PM1 towards the East where $w$ is the rotational speed of the surface of the Earth at the particular latitude. 


\subsection{Time Measurement along PM1}

The time $t_{1}(a)_{G P S}$ measured by the GPS clocks at P and M1 for the light to travel from P to $\mathrm{M} 1$ is $[19-21]$

$$
t_{1}(a)_{G P S}=\frac{l_{1}}{c}+\frac{l_{1} w}{c^{2}}
$$

while from equation (1) of ether theory

$$
t_{1}(a)=\frac{l_{1}}{c-w} \approx \frac{l_{1}}{c}+\frac{l_{1} w}{c^{2}}, w<<c
$$

Hence $t_{1}(a)_{G P S}=t_{1}(a)$ and ether drift arising from the rotation of the Earth is detected. The time $t_{1}(b)_{G P S}$ measured by the GPS clocks for the light to travel from M1 to P is [19-21]

$$
t_{1}(b)_{G P S}=\frac{l_{1}}{c}-\frac{l_{1} w}{c^{2}}
$$

while from equation (2) of ether theory

$$
t_{1}(b)=\frac{l_{1}}{c+w} \approx \frac{l_{1}}{c}-\frac{l_{1} w}{c^{2}}, w<<c
$$

Hence $t_{1}(b)_{G P S}=t_{1}(b)$ and ether drift arising from the rotation of the Earth is again detected. From ether theory as well as clock measurement, the difference in the out and back times along PM1 is given by

$$
\Delta t_{1}=t_{1}(a)-t_{1}(b)=\frac{2 l_{1}}{c} \frac{w}{c}
$$

Result (16) is first-order and therefore is not affected by second-order length contraction as is the second-order result (10) in the conventional Michelson-Morley type experiments. Equation (16) has been extensively verified in GPS operation.

\subsection{Time Measurement along PM2}

The time $t_{2}(a)_{G P S}$ for the light to travel from P to M2 measured by the GPS clocks at P and $\mathrm{M} 2$ is $[20,21]$

$$
t_{2}(a)_{G P S}=\frac{l_{2}}{c}
$$

while from equation (4) of ether theory 


$$
t_{2}(a)=\frac{l_{2}}{\sqrt{c^{2}-w^{2}}} \approx \frac{l_{2}}{c}, w<<c
$$

Hence $t_{2}(a)_{G P S}=t_{2}(a)$ and ether theory is confirmed by GPS measurement. The time $t_{2}(b)_{G P S}$ for the light to travel from M2 to P measured by the GPS clocks is [20, 21]

$$
t_{2}(b)_{G P S}=\frac{l_{2}}{c}
$$

while from equation (5) of ether theory

$$
t_{2}(b)=\frac{l_{2}}{\sqrt{c^{2}-w^{2}}} \approx \frac{l_{2}}{c}, w<<c
$$

Hence $t_{2}(b)_{G P S}=t_{2}(b)$ and ether theory is again confirmed by GPS measurement. From ether theory as well as GPS clock measurement, the difference in the out and back times along PM2 is given by

$$
\Delta t_{2}=t_{2}(a)-t_{2}(b)=0
$$

This has been confirmed by actual GPS measurements which have shown that unlike East-West travel, there is no time difference between light travelling North and light travelling South.

\section{Discussion}

The modified Michelson-Morley experiment utilizing synchronized GPS clocks to directly measure light travel times out and back along the arms of the apparatus has detected ether drift arising from the rotational motion of the Earth. The clocks have directly confirmed the light travel times for changed light speeds $c \pm w$ in the East-West direction resulting from the drift of the ether as the apparatus moves through the medium at speed $w$ corresponding to the rotational speed of the Earth's surface at the particular latitude. The time difference $\Delta t_{1}=\frac{2 l_{1}}{c} \frac{w}{c}$ between out and back light transmission in the direction of motion is first-order and is therefore immune to the effect of second-order length contraction occurring in the arm parallel to the direction of motion. The experiment is deliberately confined to the dimensions of the original Michelson-Morley apparatus where the frame is considered to be approximately inertial and where special relativity has been universally applied [22]. This is done in order to negate any objections concerning rotating coordinates and non-inertial frames which are never invoked in 
the original Michelson-Morley experiment or in any of the several modern versions of the experiment [1-10]. With the current resolution limit of GPS clocks if there is a desire to perform an actual test, the effective path length can in principle be arbitrarily increased by having repeated reflections of the light along each arm of the apparatus as is done in modern MichelsonMorley type experiments.

From the analysis it is evident that the time measurements, particularly the time difference $\Delta t_{1}=\frac{2 l_{1}}{c} \frac{w}{c}$, apply beyond the boundaries of the apparatus. Two cases involving intermediate distance travel between San Francisco and New York and long-distance travel around the Earth have been reported in the literature. Marmet [20] has discussed the case of light travel between San Francisco and New York both of which are at the same latitude with New York about $4500 \mathrm{~km}$ East of San Francisco. For these two cities $\Delta t_{1}=\frac{2 l_{1}}{c} \frac{w}{c} \approx 28 n s$ which means that light travelling from San Francisco to New York takes 28ns longer than light travelling from New York to San Francisco. Ashby [21] and Kelly [23] have discussed the case of light travel that circumnavigates the Earth along the equator. Here $\Delta t_{1}=\frac{2 l_{1}}{c} \frac{w}{c} \approx 414.8 n s$ and again this means that light travelling eastward around the equator takes $414.8 n s$ longer to circumnavigate the Earth than light travelling westward.

It should be noted that the time differences for light traveling in the East-West direction found in the modified Michelson-Morley experiment correspond to light speed variations $c \pm w$ that have been directly revealed using the synchronized clocks of the GPS [19] as well as the range equation of the GPS [24]. This appears to be a feature of light travel on the surface of the Earth as it rotates in the Earth-Centered Inertial (ECI) frame. The fact that neither the orbital or galactic motion of the Earth influences these measured light speeds suggests that the ECI is the preferred frame associated with the Earth. However Hatch [25] has argued that this behavior is an artifact of clock bias implying that the preferred frame is not necessarily carried along with the Earth as it appears. Interestingly light speed anisotropy arising from the motion of the Earth has also been reported in a laser diffraction experiment [26] and there appears to be evidence of light speed anisotropy for light propagation over cosmological distances [27]. 


\section{Conclusion}

A modified Michelson-Morley experiment employing GPS clocks instead of an interferometer for direct measurement of light travel times along the arms of the system has revealed ether drift arising from the Earth's rotation. The light travel times were directly determined and were therefore essentially immune to the second-order length contraction phenomenon, as occurs in the conventional Michelson-Morley experiments. The approach employed does not require actual time measurement but utilizes a novel feature of the GPS identified by Gift [19] where the light travel time is directly available from the CCIR clock synchronization algorithm. The modified experiment succeeds in detecting ether drift for rotational motion while all other Michelson-Morley-type experiments except the Demjanov and Galaev experiments are considered to have produced null results.

The Michelson-Gale experiment of 1925 did produce a positive result and appears to have detected ether drift associated with the Earth's rotation [28]. However the objection is sometimes raised that because of the extended dimensions of the apparatus on the rotating Earth this experiment involves rotating coordinates and non-inertial frames. Therefore light speed changes resulting in positive fringe shifts can occur without being inconsistent with the light speed invariance postulate of special relativity which applies only in inertial frames [29]. We disagree with this objection since the dimensions of the apparatus can in principle be reduced so that it occupies an approximately inertial frame while still producing a positive result using sufficiently sensitive equipment. Nevertheless in order to negate this objection in the modified Michelson-Morley experiment described in this paper, the apparatus is constrained within the boundaries of the original Michelson-Morley experiment where the frame is considered to be approximately inertial. Here special relativity is directly applicable and predicts a zero time-offlight difference between equal orthogonal arms and hence a null result [22].

Therefore the detected ether drift in the modified Michelson-Morley experiment in this approximately inertial frame is legitimate and reveals a preferred frame as previously reported by Gift [15] and Shtyrkov[16] and also by Demjanov [11] and Galaev [14]. This is consistent with the preferred frame associated with the set of "equivalent" transformations identified by Selleri [30]. This set contains all possible transformations (including the Lorentz transformation of special relativity) that connect two inertial frames under reasonable assumptions and which differ 
only by a clock synchronization parameter. With this successful detection of the ether, recent efforts at re-interpreting special relativity in the presence of a preferred frame are expected to assume greater significance as attempts to salvage this theory are undertaken [31, 32]. Also the established existence of the ether is likely to have implications for the analysis and eventual interpretation of the results of the OPERA experiment where superluminal neutrinos were reported [17].

\section{References}

1. Michelson, A.A. and E.W. Morley, The relative motion of the Earth and the luminiferous aether, Am.J.Sci., ser.3, v.34, 333-345, 1887.

2. Jaseja, T.S., A. Javan, J. Murray and C.H. Townes, Test of Special Relativity or of the Isotropy of Space by Use of Infrared Masers, Physical Review 133, A1221-A1225, March 1964.

3. Brillet, A. and J.L. Hall, Improved Laser Test of the Isotropy of Space, Physical Review Letters, 42, 549-552, February 1979.

4. Müller, H., S. Herrmann, C. Braxmaier, S. Schiller and A. Peters, Modern MichelsonMorley Experiment using Cryogenic Optical Resonators, Phys. Rev. Lett. 91, 020401, 2003.

5. Wolf, P., S. Bize, A. Clairon, G. Santarelli, M.E. Tobar and A.N. Luiten, Improved Test of Lorentz Invariance in Electrodynamics, Physical Review D 70, 051902, 2004.

6. Hermann, S., A. Senger, E. Kovalchuk, H. Müller and A. Peters, Test of the Isotropy of the Speed of Light Using a Continuously Rotating Optical Resonator, Physical Review Letters, 95, 150401, 2005.

7. Antonini, P., M. Okhapkin, E. Goklu, E. and S. Schiller, Test of Constancy of Speed of Light With Rotating Cryogenic Optical Resonators, Physical Review A 71, 050101, 2005.

8. Müller, H., P.L. Stanwix, M.E. Tobar, E. Ivanov, P. Wolf, S. Herrmann, A. Senger, E. Kovalchuk and A. Peters, "Relativity tests by complementary rotating Michelson-Morley experiments". Phys. Rev. Lett. 99 (5): 050401, 2007. 
9. Eisele, C, A. Nevsky and S. Schiller, Laboratory Test of the Isotropy of Light Propagation at the 10 $0^{-17}$ Level, Physical Review Letters, 103, 090401, 2009.

10. Herrmann, S., A. Senger, K. Mohle, M. Nagel, E. V. Kovalchuk and A. Peters, Rotating Optical Cavity Experiment Testing Lorentz Invariance at the $10^{-17}$ Level, Physical Review D 80, 105011, 2009.

11. Demjanov, V.V., Physical interpretation of the fringe shift measured on Michelson interferometer in optical media. Physics Letters A 374, 1110-1112, 2010.

12. Demjanov, V.V., What and how does a Michelson interferometer measure? arXiv:1003.2899v4 [physics.gen-ph] 18 January 2011.

13. Demjanov, V.V., Michelson Interferometer Operating at Effects of First Order with Respect to v/c (the third method of measuring the speed of "aether wind"), arXiv:quanth/0103103v3 19 April 2010.

14. Galaev, Y.M., The Measuring of Ether-Drift Velocity and Kinematic Ether Viscosity Within Optical Wavebands, Spacetime \& Substance 3 (5,15) (2002) p207-224

15. Gift, S.J.G., The Relative Motion of the Earth and the Ether Detected, Journal of Scientific Exploration, 20, 201, 2006.

16. Shtyrkov, E.I., Observation of Ether Drift in Experiments with Geostationary Satellites, Proceedings of the Natural Philosophy Alliance, pp201-205, 12 $2^{\text {th }}$ Annual Conference, Storrs CT, 23-27, May 2005.

17. The OPERA Collaboration, Measurement of the neutrino velocity with the OPERA detector in the CNGS beam, arXiv:1109.4897, 23 September 2011.

18. Sardin, G., Testing Lorentz symmetry of special relativity by means of the Virgo or Ligo set-up, through the differential measure of the two orthogonal beams time-of-flight, arXiv:physics/0404116v1 [physics.gen-ph] 25 April 2004

19. Gift, S.J.G., One-Way Light Speed Measurement Using the Synchronized Clocks of the Global Positioning System (GPS), Physics Essays, 23, 271, 2010.

20. Marmet, P., The GPS and the Constant Velocity of Light, Acta Scientiarum, 22, 1269, 2000.

21. Ashby, N., Relativity in the Global Positioning System, Living Reviews in Relativity, 6, $1,2003$. 
22. Rindler, W., Relativity Special, General and Cosmological, $2^{\text {nd }}$ edition, Oxford University Press, New York, 2006.

23. Kelly, A., Challenging Modern Physics, BrownWalker Press, Florida, 2005.

24. Gift, S.J.G., One-Way Light Speed Determination Using the Range Measurement Equation of the GPS, Applied Physics Research, 3, 110, 2011.

25. Hatch, R.R., Those Scandalous Clocks, GPS Solutions, 8, 67, 2004.

26. Navia,C.E., C.R. Augusto, D.F. Franceschini, M.B. Robba and K.H. Tsui, Seach for Anisotropic Light Propagation as a Function of Laser Beam Alignment Relative to the Earth's Velocity Vector, Progress in Physics, Vol.1, January 2007, pp53-60.

27. Nodland,B. and J.P. Ralston, Indication of Anisotropy in Electromagnetic Propagation over Cosmological Distances, Physical Review Letters, 78, 3043, 1997.

28. Michelson, A.A., and H.E. Gale, The Effect of the Earth's Rotation on the Velocity of Light, Astrophysical Journal, 61, 140, 1925.

29. Brown, K., Reflections on Relativity, Lulu.com, 2011; also at http://www.mathpages.com/rr/rrtoc.htm

30. Selleri, F., Recovering the Lorentz Ether, Apeiron, 11, 246, 2004.

31. Guerra, V. and R. de Abreu, On the Consistency Between the Assumption of a Special System of Reference and Special Relativity, Foundations of Physics, 36, 1826, 2006.

32. Perez, I., The Physics Surrounding the Michelson-Morley Experiment and a New Aether Theory, arXiv:1004.0716v3 [physics.gen-ph] 15 Dec 2010. 